\title{
Gamma Knife surgery for central neurocytomas
}

\author{
Clinical article
}

\author{
Bengt Karlsson, M.D., Ph.D., ${ }^{1}$ Wan-Yuo Guo, M.D., Ph.D., ${ }^{2}$ Teo Kejia, M.B.B.S., ${ }^{1}$ \\ Nivedh Dinesh, ${ }^{1}$ David Hung-Chi Pan, M.D., ${ }^{2}$ Hidefumi Jokura, M.D., ${ }^{3}$ \\ Jun Kawagishi, M.D., ${ }^{3}$ Albertus T. C. J. van Eck, M.D., ${ }^{5}$ Gerhard A. Horstmann, M.D., 5 \\ Tseng Tsai YeO, M.B.B.S., F.R.A.C.S., ${ }^{1}$ and Masaaki Yamamoto, M.D. ${ }^{4}$
}

${ }^{1}$ Division of Neurosurgery, National University Hospital, Singapore; ${ }^{2}$ Veterans General Hospital, Taipei, Taiwan; ${ }^{3}$ Jiro Suzuki Memorial Gamma House, Furukawa Seiryo Hospital, Furukawa, Japan; ${ }^{4}$ Katsuta Hospital Mito Gamma House, Ibaraki, Japan; and ${ }^{5}$ Krefeld Gamma Knife Center, Krefeld, Germany

\begin{abstract}
Object. The optimal management of central neurocytoma $(\mathrm{CN})$ remnants and recurrences is still not clear. To date no large series of patients treated with Gamma Knife surgery (GKS) for CNs has been published. For that reason the authors decided to combine data from 5 different centers so that they could analyze the largest population of patients treated with GKS for CN currently available.

Methods. Data obtained in 42 patients who were treated for CN with GKS before July 1, 2010, were retrospectively collected and analyzed. The median prescribed dose was $13 \mathrm{~Gy}$ (range 11-25 Gy). The follow-up time in these patients ranged from 0.5 to 14.7 years (mean 6.1 years, median 4.9 years). Eleven patients were followed up for 5-10 years and 9 patients for more than 10 years. All patients were alive and well at the closing of the study except 1 patient, who died of injuries sustained in a traffic accident.

Results. Two cases of local tumor progression and 2 cases of distant tumor recurrence occurred among the patient population, yielding 5- and 10-year tumor control rates of $91 \%$ and $81 \%$, respectively. No permanent complications occurred. The findings were in line with results reported in earlier publications. Despite the high tumor control rate, enlargement of part of or the whole ventricular system was seen in $45 \%$ of patients.

Conclusions. The high tumor control rate and the low complication rate following GKS indicate that GKS is the preferred treatment for $\mathrm{CN}$ tumor remnants or recurrences following microsurgery. However, data from longer follow-up times in more patients are needed before this conclusion can be validated. The patients need to be closely monitored and potential hydrocephalus managed despite tumor control.

(http://thejns.org/doi/abs/10.3171/2012.6.GKS12214)
\end{abstract}

\section{KeY Words • Gamma Knife surgery stereotactic radiosurgery}

$\mathrm{C}$ ENTRAL neurocytomas are neuronal tumors that most commonly arise from the wall of the lateral ventricle. Histologically, these lesions are typically benign, although atypical tumors have also been reported. ${ }^{26}$ Central neurocytomas were first described in 1982, when Hassoun et al. ${ }^{8}$ reported 2 tumors that "highly calcified, were composed of small clear cells forming dense areas in a patchy fibrillary stroma. Electron microscopy revealed a striking neuronal differentiation with numerous synapses."

Central neurocytomas are usually diagnosed when there is blockage of the CSF pathways, causing hydrocephalus and elevated intracranial pressure. Microsurgical removal is the treatment of choice. ${ }^{12}$ The results of microsurgical removal are dependent on the extent of the resection and the histological features of the tumor. ${ }^{14,21,24}$ Total resection and an MIB-1 index of $<2-3$ indicate a high likelihood for local tumor control. ${ }^{14,24}$ Correspondingly, the risk for tumor recurrence is higher in patients in whom subtotal tumor resection is performed or in whom the tumor is histopathologically atypical.

Abbreviations used in this paper: $\mathrm{CN}=$ central neurocytoma; GKS = Gamma Knife surgery.
- central neurocytoma • radiation •

Fractionated radiotherapy results in an increased likelihood of tumor control in cases of subtotal tumor removal or lesions with atypical histological characteristics. ${ }^{14,22,24}$ However, severe long-term complications have been reported following radiotherapy. ${ }^{19}$ Multiple reports suggest that GKS may result in a high rate of tumor control and a low complication rate, making it conceptually an attractive alternative to radiotherapy. ${ }^{1-4,6,7,9,10,16,20,25,27}$

Chen et al. ${ }^{3}$ reported $100 \%$ tumor control and no complications in 14 patients following GKS for $\mathrm{CN}$ and a median follow-up lasting more than 5 years. However, those authors thought that their documentation was not strong enough to be conclusive and recommended that a multicenter study be conducted with more patients and a longer follow-up time to confirm their results. We therefore decided to add data from 4 additional centers to updated information on the patient population described in the report by Chen et al.

\section{Methods}

All patients who harbored CNs that were treated with GKS before July 1, 2010, in the participating institutions were eligible for the study. Most patients (21) were treated 
at Veterans General Hospital, Taipei, Taiwan. In addition to these, 8 patients were treated at Jiro Suzuki Memorial Gamma House, Furukawa Seiryo Hospital, Furukawa, Japan; 7 patients at Katsuta Hospital Mito Gamma House, Ibaraki, Japan; 5 patients at the Singapore Gamma Knife Center, Singapore; and 2 patients at Gamma Knife Zentrum Krefeld, Germany. Excluded was 1 patient in whom no follow-up information was available. In total, 42 eligible patients were included in the study. Informed consent was obtained from the patients when possible, and approval of the study was granted by the ethics committees of all participating institutions.

The tumors included recurrent lesions after gross total removal in 2 patients and tumor remnants after subtotal removal in 33 patients. A biopsy was performed in 1 patient, and the diagnosis was based on imaging studies in 6 patients. All 36 tumors for which histological results were available were benign. There was evidence of tumor growth between microsurgery and GKS in the 2 patients who underwent gross total tumor removal and in 9 of the 33 patients in whom partial tumor removal was performed. One patient had received prior radiotherapy.

The patients in this study ranged in age from 4 to 71 years (mean 32 years, median 31 years). Twenty-four patients were male $(57 \%)$ and 18 were female $(43 \%)$. The tumor volumes ranged from 1 to $49 \mathrm{~cm}^{3}$ (mean $12 \mathrm{~cm}^{3}$, median $8 \mathrm{~cm}^{3}$ ), and the prescribed radiation doses ranged from 11 to $25 \mathrm{~Gy}$ (mean $14 \mathrm{~Gy}$, median $13 \mathrm{~Gy}$ ). There was a correlation between the prescribed dose and tumor volume: larger tumors were given lower radiation doses $(\mathrm{p}=$ 0.036). All but 1 patient, who died of injuries sustained in a traffic accident, were alive and well at the time the study was closed.

The end points in the study were tumor growth or tumor removal by microsurgery. Tumor control was defined as no growth of the treated tumor as well as absence of distant recurrence. For the purposes of this report, a lack of local tumor control as well as a distant recurrence are defined as tumor recurrence. Time at risk for tumor growth is defined as the time interval between GKS and the first of the following events: local tumor growth (2 patients) or distant tumor recurrence (2 patients); tumor removal due to ventricle enlargement (2 patients); death (1 patient); and latest imaging follow-up examination (35 patients). Clinical follow-up ranged from 0.5 to 14.7 years (mean 6.1 years, median 4.9 years).

The latest follow-up information for the 35 patients still at risk for tumor recurrence is presented here. The year of the latest imaging study was 2012 in 2 patients, 2011 in 21 patients, 2010 in 9 patients, and 2009 in 3 patients. Eight patients were followed up for more than 10 years and another 10 patients for 5-10 years. The interval between GKS and the latest imaging examination ranged from 1.4 to 14.1 years (median 5.0 years, mean 6.2 years), and the latest clinical data were collected between 1.4 and 14.7 years (median 5.0 years, mean 6.4 years).

\section{Statistical Analysis}

Kaplan-Meier survival statistics were used to analyze time free from tumor recurrence, and a log rank (MantelCox) test was performed to compare 2-group variables using survival statistics. The Mann-Whitney U-test was used to compare nominal and continuous data, and the Wilcoxon signed rank test was used to analyze the correlation between paired continuous data. The Fisher exact test was used for nominal data. A difference was considered statistically significant when $\mathrm{p}<0.05$.

\section{Results}

\section{Tumor Control Rate Following GKS}

Four cases of tumor recurrence occurred in the patient population: 2 recurrent tumors were local (2.4 and 9.6 years after GKS) and 2 were distant (1.6 and 4.8 years after GKS). Thus, the 5- and 10-year tumor control rates were $91 \%$ and $81 \%$, respectively (Fig. 1). The local tumor control rate was unrelated to the prescribed dose $(\leq 13$ vs $>13 \mathrm{~Gy}, \mathrm{p}=0.95)$ and tumor volume $(<7.55$ vs $>7.55$ $\mathrm{cm}^{3}, \mathrm{p}=0.83$ ). Patients who underwent partial or total tumor removal had a higher chance of local tumor control than patients who did not undergo surgery $(\mathrm{p}=0.025)$; however, no statistical significance was found when prior surgery or not was compared with tumor recurrence (local or distant $)(\mathrm{p}=0.32)$.

The fact that only 2 cases of local tumor recurrence were observed limits the value of the statistical analyses.

\section{Ventricle Size}

An increase in part of or the whole ventricular system (hereafter referred to as ventricle enlargement) occurred in 19 patients (45\%) after GKS. The frontal or occipital horns increased in size in 5 patients, an enlargement in a cystic component of the ventricular system occurred in 3 patients, and a general increase in ventricle size in 11 patients - one of whose imaging studies are presented in Fig. 2. The ventricle enlargement prompted surgical intervention in 6 patients $(32 \%)$. Tumors were removed in 2 patients because of ventricle enlargement despite tumor control. A shunt was inserted in 1 patient, and shunt revisions were performed in 2 patients. Aspiration from an Omaya reservoir was performed in 1 patient. Two additional patients had hydrocephalus before GKS. The assumption was that the hydrocephalus should normalize after GKS. This did not happen, and both patients received shunts following GKS.

Using survival statistics, we found a statistically significant relationship between ventricle enlargement and radiation dose ( $\leq 13$ vs $>13$ Gy; $p=0.0086)$. No significant relationship with ventricle enlargement was found for tumor size $\left(<7.55\right.$ or $\left.>7.55 \mathrm{~cm}^{3} ; \mathrm{p}=0.054\right)$, whether there was prior surgery $(p=0.63)$, or whether there was tumor control $(\mathrm{p}=0.91)$. The results changed when the MannWhitney U-test or Fisher exact test was used instead of survival statistics. Using the Mann-Whitney U-test, the relationship between tumor size and ventricle enlargement was found to be significant ( $p=0.0043$ ) but not the treatment dose $(p=0.11)$. Using the Fischer exact test, no significant relationship could be found between ventricle enlargement and previous surgery $(p=0.43)$ or whether tumor control had been achieved or not $(\mathrm{p}=0.61)$. 


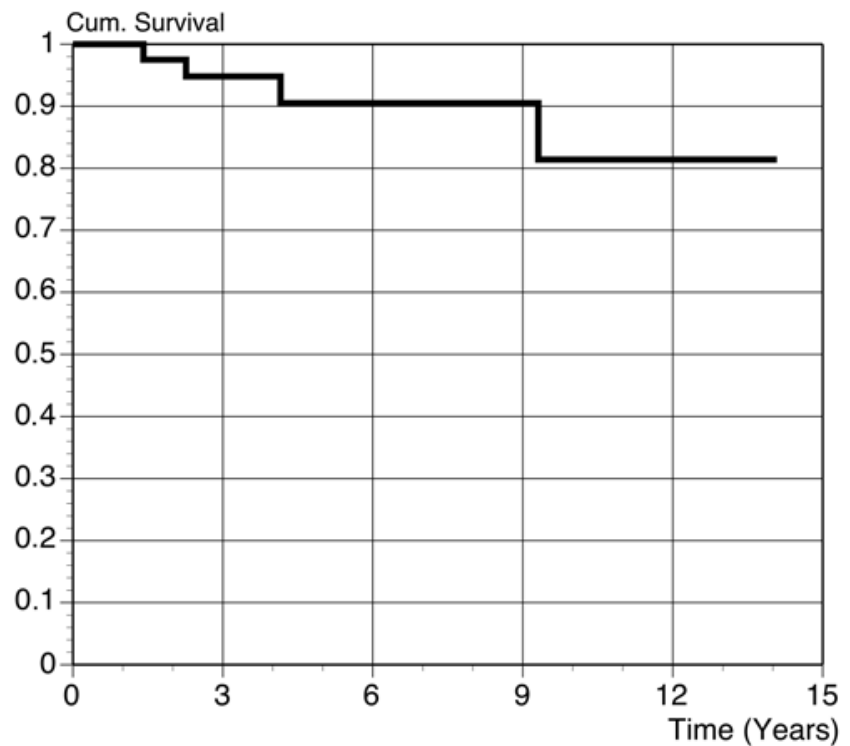

FIG. 1. Kaplan-Meier plot illustrating the time of freedom from tumor recurrence following GKS in years. Cum. = Cumulative.

\section{Clinical Outcome Following GKS}

In 1 patient a symptomatic edema developed close to the target volume posterior to the left occipital horn. The edema developed parallel to an increase in the size of the ventricle horn (Fig. 3), and edema as well as symptoms disappeared simultaneously when the ventricle horn was reduced to normal size. No other symptoms, besides those caused by hydrocephalus as mentioned earlier, developed in the patient population. All but 1 patient, who died of injuries sustained in a traffic accident, were alive and well at the time the study was closed.

\section{Management of Tumor Recurrences}

One of the 2 patients with local tumor recurrence was treated again with GKS; the other continues to be monitored 22 months after diagnosis of recurrent tumor because there are still no symptoms. One of the 2 patients who harbored a distant recurrence was retreated with GKS; the other was scheduled to undergo fractionated radiotherapy but declined treatment. Follow-up times are still too short to assess the results of repeated treatment. Not using tumor removal in the management of tumor recurrences in our patient population is in line with earlier published data, in which 4 of 5 local recurrent tumors were retreated with GKS; management of the fifth recurrent tumor was not reported..$^{10,16,27}$

\section{Discussion}

\section{Histological Characteristics of CNs}

Central neurocytomas were initially defined as benign tumors, and thus the prognosis was deemed good following total tumor removal. Today we know that this definition does not apply to all CNs. A less favorable prognosis has been reported in tumors with a high MIB-1 index or atypical histological characteristics. ${ }^{14,21}$ This must
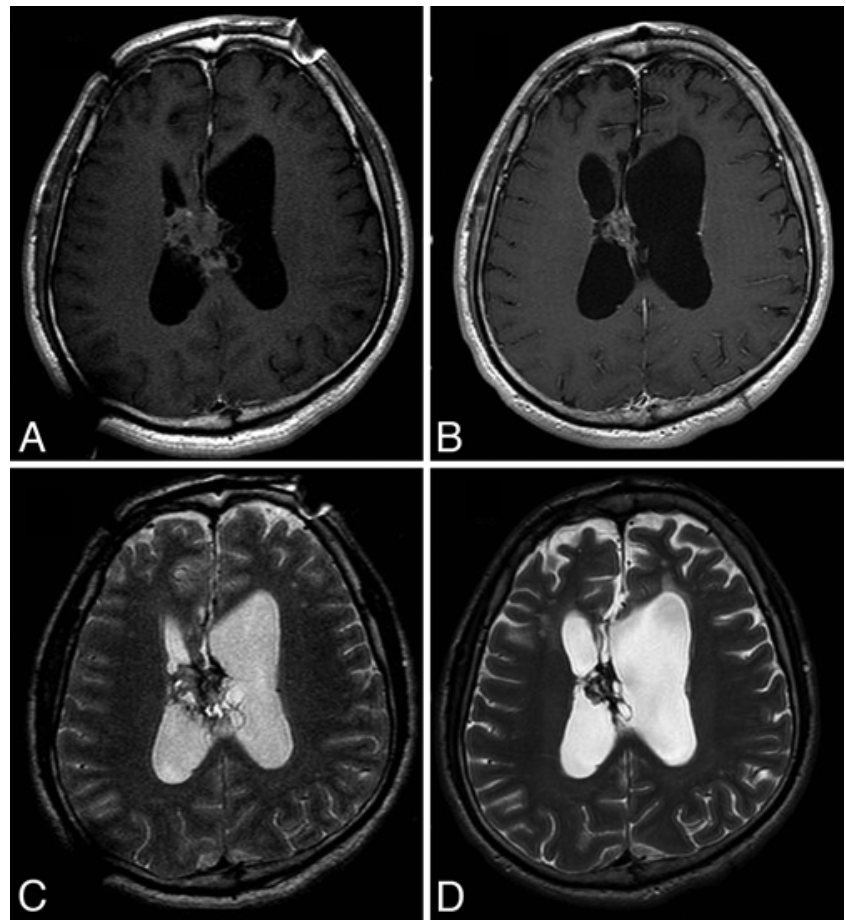

FIG. 2. Magnetic resonance images obtained at the time GKS was performed ( $A$ and $\mathbf{C})$ and 10 years after GKS (B and D). The T1weighted images shown in panels $A$ and $B$ reveal a significant decrease in tumor volume, whereas the lower images (C and $D)$ show an increase in ventricle size despite shrinkage of the tumor.

be taken into account when comparing the results in the present study to those from other studies. To extrapolate our results to what can be expected should atypical tumors be treated with GKS would be a questionable move. Genc et al. ${ }^{6}$ reported results following GKS for $18 \mathrm{CNs}$ of which 3 had an MIB-1 index $\geq 4$. Tumor control was reported in all but 1 case, in which there was recurrence of a tumor with an MIB-1 index of 4. Thus, it is possible that GKS will yield less favorably outcomes when atypical CNs are treated.

\section{Including CNs Based on Imaging Only}

The imaging features of a $\mathrm{CN}$ are quite characteristic. On MR images, this tumor usually presents as an intraventricular, circumscribed, lobular, and "bubbly" mass lesion. In most cases, it is attached to the septum pellucidum, may cause an obstruction of the cerebrospinal fluid circulation at the level of the foramen of Monro, and results in an isolated dilated ventricle containing highprotein fluid. Magnetic resonance imaging also displays moderate-to-strong heterogeneous enhancement following administration of gadolinium. On MR spectroscopic images, a glycine peak at $3.55 \mathrm{ppm}$ is typically present.

We concur with the opinion that histological verification of CNs should be recommended, but we also believe that it is fair to treat patients with CNs based on imaging evidence alone in cases in which there are contraindications to open surgery. There was only imaging evidence and no histological verification in 6 of the 42 tumors included in this series. We have just stated one rationale 

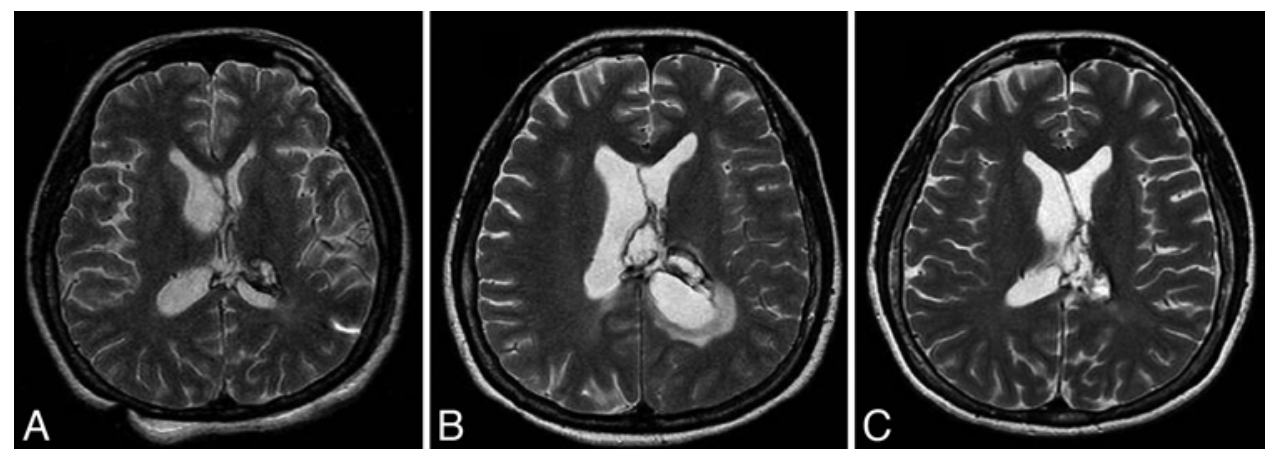

FIG. 3. Magnetic resonance T2-weighted images obtained on the day of GKS (A) and 6 months later (B). A significant increase in the size of the left occipital horn has developed, as has surrounding edema. A shunt revision was performed. The situation normalized and the edema disappeared, as shown on an image obtained 9 years after GKS (C).

not to exclude treating these lesions. Another is that most vestibular schwannomas and meningiomas included in radiosurgical reports lack histological verification of the type of lesion. An additional argument is that including these 6 cases enabled us to analyze the impact of surgery on the incidence of ventricle enlargement following GKS The most compelling argument for us, however, is that excluding these 6 cases would have changed the results of the study. With them, the 10-year tumor control rate was $81 \%$, and the median radiological follow-up time was 4.8 years. Without these 6 patients, the respective numbers would have been $83 \%$ and 5.0 years. We therefore believe that our results were made more representative of $\mathrm{CN}$ cases by including these 6 patients.

\section{Enlargement of the Ventricular System Following GKS}

The relationship between ventricle enlargement and tumor and treatment parameters should be analyzed using survival statistics if we assume that the likelihood for ventricle enlargement increases with time and that an increase is irreversible. Other statistical methods should be used if this is not the case.

We found a statistically significant relationship between treatment dose and the incidence of ventricle enlargement using survival statistics. This significance disappeared when we used the Mann-Whitney U-test; instead the relationship between tumor volume and ventricle enlargement became statistically significant. Our interpretation of the findings is that larger tumor volumes result in a higher incidence of post-GKS ventricle enlargement, and the observation that tumors treated with lower radiation doses have a higher incidence of ventricle enlargement is due to the fact that lower doses were given to larger tumors.

No treatment was deemed necessary for the majority of patients in whom ventricle enlargement developed following GKS. This and the generally uncomplicated management of hydrocephalus are probably reasons why ventricle enlargement has not been addressed in earlier publications. We believe that our observations may be of value. Ventricle size should be monitored even in patients with tumor control. An impairment of the cerebrospinal fluid circulation may lead to serious consequences if left untreated. An example of this was reported by Kulkarni et al. ${ }^{13}: 1$ of their 8 patients "died 5 years after treatment from shunt dysfunction."

\section{Comparison of the Results of This Study and Other Published Data}

The results from 12 earlier published studies are listed in Table $1.1,2,4,6,7,10,11,15,16,20,25,27$ Six tumor recurrences (1 local and 5 distant) and no complications were reported among 68 patients, yielding an approximate tumor control rate of $93 \%$. These results are comparable to ours; in our study there were no permanent complications and 4 recurrent tumors ( 2 local and 2 distant) in 42 patients $(38 / 42=90 \%)$.

\section{Results of Radiotherapy for $\mathrm{CN}$}

Radiotherapy is conceptually a more appealing treatment choice if suspicion is high that tumor cells may be present outside the visible tumor volume. The literature reporting results following radiotherapy is, however, quite sparse. A Medline search performed using the search term "neurocytoma" resulted in 544 references. The titles of these references indicated that the results of radiotherapy for $\mathrm{CN}$ were addressed in only 5 of these publications..$^{12,13,17-19}$ Two articles were from the same institution, and it is thus likely that some patients were included in both papers. ${ }^{12,19}$ Consequently, we only included the results from the latest of these 2 articles in Table 2. Tumor control was reported in 16 of 17 patients-a tumor control rate similar to that achieved by GKS. Rades et al. ${ }^{23}$ added data from 78 patients with CNs reported in the literature to 11 patients treated at their own institution, yielding 89 patients. We have been unable to trace most of the publications in which the additional cases were reported. The patient population was divided into low-dose $(<54 \mathrm{~Gy})$ and high-dose ( $\geq 54 \mathrm{~Gy}$ ) groups. The 10-year local tumor control rate was $65 \%$ for the low-dose group and $89 \%$ for the high-dose group, indicating that higher radiotherapy doses are needed to achieve tumor control.

The important difference between radiotherapy and GKS seems to be the risk of complications. Paek et al. ${ }^{19}$ reported results for 6 patients who were treated with radiotherapy (median dose $54 \mathrm{~Gy}$ ) and followed up for more than 10 years. Tumor control was observed in all patients, but radiation-induced complications developed in half of 
TABLE 1: Summary of cases of CN treated by radiosurgery*

\begin{tabular}{|c|c|c|c|c|c|c|c|c|c|c|}
\hline \multirow[b]{2}{*}{ Authors \& Year } & \multirow[b]{2}{*}{ Treatment } & \multirow[b]{2}{*}{ No. of Patients } & \multicolumn{3}{|c|}{ Radiation Dose (Gy) } & \multicolumn{3}{|c|}{ Follow-Up (yrs) } & \multirow{2}{*}{$\begin{array}{l}\text { No. of Patients w/ } \\
\text { Tumor Control (\%) }\end{array}$} & \multirow[b]{2}{*}{ No. of Complications } \\
\hline & & & Low & High & Mean & Shortest & Longest & Median & & \\
\hline Anderson et al., 2001 & GKS & 4 & 16 & 20 & 17 & 1.0 & 2.3 & 1.4 & $4(100)$ & 0 \\
\hline Cobery et al., 2001 & GKS & 4 & 9 & 13 & 11 & 1.0 & 8.3 & 3.7 & $4(100)$ & 0 \\
\hline Genc et al., 2011 & GKS & 18 & 12 & 22 & 16 & 0.5 & 9.2 & 3.0 & $17(94)$ & 0 \\
\hline Kim et al., 2007 & GKS & 13 & 9 & 20 & 16 & 0.5 & 8.0 & 5.1 & $11(85)$ & 0 \\
\hline Martín et al., 2003 & LINAC & 4 & 16 & 18 & 17 & 0.3 & 4.5 & 2.8 & $4(100)$ & 0 \\
\hline Matsunaga et al., 2010 & GKS & 8 & 12 & 18 & 14 & 1.3 & 11.3 & 5.3 & $7(88)$ & 0 \\
\hline Pollock \& Stafford, 2001 & GKS & 1 & 18 & 18 & 18 & 2.8 & 2.8 & 2.8 & $1(100)$ & 0 \\
\hline Tyler-Kabara et al., 2001 & GKS & 4 & 14 & 20 & 16 & 1.5 & 3.5 & 2.8 & $4(100)$ & 0 \\
\hline
\end{tabular}

* Data from the article by Chen et al. ${ }^{3}$ are excluded because their patients are included with ours in this paper. Abbreviation: LINAC = linear accelerator.

them. In addition, 1 patient was found to harbor an atypical meningioma that may have been radiation induced. In this group of patients, 1 complication was lethal and the other 2 resulted in white-matter degeneration and slow deteriorations in patient performance. This complication rate seems to be higher than the $5 \%$ risk of severe toxicity at 5 years that was estimated by Emami et al. ${ }^{5}$ when one-third of the brain was given $60 \mathrm{~Gy}$. The fact that no radiation-induced complication occurred among the 9 patients in our patient population observed for more than 10 years strongly suggests that the risk for radiation-induced complications is lower following GKS than it is following radiotherapy.

\section{Tumor Control After GKS}

Tumor control following GKS denotes shrinkage or no growth of the irradiated tumor, as shown in Fig. 2. In contrast, failed tumor control is generally defined as an increase in the volume of the irradiated tumor or a growth of tumor contiguous with the treated tumor. Tumor growth found elsewhere is often referred to as a distant or out-of-field recurrence. The distinction between local and distant recurrences is logical when analyzing the tumor response to radiosurgery. It is, however, less logical from a clinical standpoint, because in both cases the intracerebral disease is uncontrolled.
One can argue that local tumor control reflects the efficacy of GKS, whereas distant tumor control is more related to patient selection. A distant recurrence implies that tumor cells were left untreated at the time of GKS, and thus a targeted volume larger than the visible tumor volume may have been advantageous for the patient. Would radiotherapy thus have been a better treatment option for these patients? Based on the findings we offered earlier, the tumor control rate following radiotherapy appears similar to that following GKS, and it seems that a low incidence of distant recurrences does not justify a higher risk for long-term complications following radiotherapy. In addition, distant tumor recurrence appears to be treatable with radiation in the majority of the cases, making a recurrent tumor manageable.

\section{Conclusions}

The tumor control rate following GKS is high, and the risk-benefit relationship compares favorably to that following fractionated radiotherapy of $\mathrm{CN}$, even when low prescribed doses are used. As the long-term complication rate following fractionated radiotherapy seems to be significantly higher than that following GKS, it seems that GKS qualifies as the radiation treatment of choice for well-defined tumor remnants after $\mathrm{CN}$ surgery. The

TABLE 2: Summary of cases of CN treated by radiotherapy*

\begin{tabular}{|c|c|c|c|c|c|c|c|c|c|}
\hline \multirow[b]{2}{*}{ Authors \& Year } & \multirow[b]{2}{*}{ No. of Patients } & \multirow[b]{2}{*}{ Dose per Fraction } & \multicolumn{2}{|c|}{$\begin{array}{l}\text { Radiation } \\
\text { Dose (Gy) }\end{array}$} & \multicolumn{3}{|c|}{ Follow-Up (yrs) } & \multirow{2}{*}{$\begin{array}{l}\text { No. of Patients w/ } \\
\text { Tumor Control (\%) }\end{array}$} & \multirow[b]{2}{*}{ No. of Complications } \\
\hline & & & Min & Max & Shortest & Longest & Mean & & \\
\hline Nakagawa et al., 1993 & 2 & NR & 58 & 60 & 2.5 & 9.5 & 6.0 & $2(100)$ & 0 \\
\hline Namiki et al., 1998 & 1 & NR & 70 & 70 & 15.0 & 15.0 & 15.0 & $1(100)$ & 0 \\
\hline
\end{tabular}

${ }^{*} \mathrm{NR}=$ not reported. 
fact that GKS is presently the best documented radiation treatment supports this conclusion. Local and distant tumor recurrences can be favorably managed using additional radiation treatment. The size of the ventricle needs to be monitored following GKS, because an enlargement of the ventricular system, or parts of it, may occur even in patients in whom the tumor is controlled. Ventricle enlargement among these patients sometimes necessitates surgical intervention.

\section{Acknowledgment}

The authors thank Dr. Ho Kee Han for sharing his data.

\section{Disclosure}

Professor Karlsson is a consultant to Elekta AB.

Author contributions to the study and manuscript preparation include the following. Conception and design: all authors. Acquisition of data: all authors. Analysis and interpretation of data: Karlsson, Guo, Yeo. Drafting the article: Karlsson, Dinesh, Jokura, Yeo, Yamamoto. Critically revising the article: all authors. Reviewed submitted version of manuscript: all authors. Approved the final version of the manuscript on behalf of all authors: Karlsson. Statistical analysis: Karlsson.

\section{References}

1. Anderson RC, Elder JB, Parsa AT, Issacson SR, Sisti MB: Radiosurgery for the treatment of recurrent central neurocytomas. Neurosurgery 48:1231-1238, 2001

2. Bertalanffy A, Roessler K, Dietrich W, Aichholzer M, Prayer D, Ertl A, et al: Gamma knife radiosurgery of recurrent central neurocytomas: a preliminary report. J Neurol Neurosurg Psychiatry 70:489-493, 2001

3. Chen MC, Pan DH, Chung WY, Liu KD, Yen YS, Chen MT, et al: Gamma knife radiosurgery for central neurocytoma: retrospective analysis of fourteen cases with a median followup period of sixty-five months. Stereotact Funct Neurosurg 89:185-193, 2011

4. Cobery ST, Noren G, Friehs GM, Chougule P, Zheng Z, Epstein $\mathrm{MH}$, et al: Gamma knife surgery for treatment of central neurocytomas. Report of four cases. J Neurosurg 94: 327-330, 2001

5. Emami B, Lyman J, Brown A, Coia L, Goitein M, Munzenrider JE, et al: Tolerance of normal tissue to therapeutic irradiation. Int J Radiat Oncol Biol Phys 21:109-122, 1991

6. Genc A, Bozkurt SU, Karabagli P, Seker A, Bayri Y, Konya D, et al: Gamma knife radiosurgery for cranial neurocytomas. J Neurooncol 105:647-657, 2011

7. Hara M, Aoyagi M, Yamamoto M, Maehara T, Takada Y, Nojiri T, et al: Rapid shrinkage of remnant central neurocytoma after gamma knife radiosurgery: a case report. J Neurooncol 62:269-273, 2003

8. Hassoun J, Gambarelli D, Grisoli F, Pellet W, Salamon G, Pellissier JF, et al: Central neurocytoma. An electron-microscopic study of two cases. Acta Neuropathol 56:151-156, 1982

9. Javedan SP, Manwaring K, Smith KA: Treatment of posterior third ventricular central neurocy toma with endoscopic biopsy, endoscopic third ventriculostomy and stereotactic radiosurgery. Minim Invasive Neurosurg 46:165-168, 2003

10. Kim CY, Paek SH, Jeong SS, Chung HT, Han JH, Park CK, et al: Gamma knife radiosurgery for central neurocytoma: primary and secondary treatment. Cancer 110:2276-2284, 2007

11. Kim CY, Paek SH, Kim DG: Linear accelerator radiosurgery for central neurocytoma: a case report. J Neurooncol 61:249254,2003
12. Kim DG, Paek SH, Kim IH, Chi JG, Jung HW, Han DH, et al: Central neurocytoma: the role of radiation therapy and long term outcome. Cancer 79:1995-2002, 1997

13. Kulkarni V, Rajshekhar V, Haran RP, Chandi SM: Long-term outcome in patients with central neurocytoma following stereotactic biopsy and radiation therapy. Br J Neurosurg 16: 126-132, 2002

14. Leenstra JL, Rodriguez FJ, Frechette CM, Giannini C, Stafford SL, Pollock BE, et al: Central neurocytoma: management recommendations based on a 35 -year experience. Int J Radiat Oncol Biol Phys 67:1145-1154, 2007

15. Martín JM, Katati M, López E, Bullejos JA, Arregui G, Busquier H, et al: Linear accelerator radiosurgery in treatment of central neurocytomas. Acta Neurochir (Wien) 145:749_ 754,2003

16. Matsunaga S, Shuto T, Suenaga J, Inomori S, Fujino H: Gamma knife radiosurgery for central neurocytomas. Neurol Med Chir (Tokyo) 50:107-113, 2010

17. Nakagawa K, Aoki Y, Sakata K, Sasaki Y, Matsutani M, Akanuma A: Radiation therapy of well-differentiated neuroblastoma and central neurocytoma. Cancer 72:1350-1355, 1993

18. Namiki J, Nakatsukasa M, Murase I, Yamazaki K: Central neurocytoma presenting with intratumoral hemorrhage 15 years after initial treatment by partial removal and irradiation. Neurol Med Chir (Tokyo) 38:278-282, 1998

19. Paek SH, Han JH, Kim JW, Park CK, Jung HW, Park SH, et al: Long-term outcome of conventional radiation therapy for central neurocytoma. J Neurooncol 90:25-30, 2008

20. Pollock BE, Stafford SL: Stereotactic radiosurgery for recurrent central neurocytoma: case report. Neurosurgery 48: 441-443, 2001

21. Rades D, Fehlauer F, Schild SE: Treatment of atypical neurocytomas. Cancer 100:814-817, 2004

22. Rades D, Schild SE, Fehlauer F: Defining the best available treatment for neurocytomas in children. Cancer 101:26292632, 2004

23. Rades D, Schild SE, Ikezaki K, Fehlauer F: Defining the optimal dose of radiation after incomplete resection of central neurocytomas. Int J Radiat Oncol Biol Phys 55:373-377, 2003

24. Schild SE, Scheithauer BW, Haddock MG, Schiff D, Burger PC, Wong WW, et al: Central neurocytomas. Cancer 79:790795, 1997

25. Tyler-Kabara E, Kondziolka D, Flickinger JC, Lunsford LD: Stereotactic radiosurgery for residual neurocytoma. Report of four cases. J Neurosurg 95:879-882, 2001

26. Yaşargil MG, von Ammon K, von Deimling A, Valavanis A, Wichmann W, Wiestler OD: Central neurocytoma: histopathological variants and therapeutic approaches. J Neurosurg 76:32-37, 1992

27. Yen CP, Sheehan J, Patterson G, Steiner L: Gamma knife surgery for neurocytoma. J Neurosurg 107:7-12, 2007

\footnotetext{
Manuscript submitted January 28, 2012.

Accepted June 5, 2012.

Findings from this study were presented at the Annual Scientific Meeting of the Neurosurgical Society of Australasia, Nadi, Fiji, September 21-24, 2011, and the 6th Saudi Association of Neurological Surgeons Annual Meeting, Al Khobar, Saudi Arabia, April 3-5, 2012.

Please include this information when citing this paper: DOI: 10.3171/2012.6.GKS12214.

Address correspondence to: Bengt Karlsson, M.D., Ph.D., Division of Neurosurgery, National University Hospital, 5 Lower Kent Ridge Road, Singapore 119074. email: nykuttram@yahoo.se.
} 